IX.

Das Rothholz aus den Fabriken des Vereins fur chemische Industrie in Mainz.

$$
\text { Von }
$$

R. Fresenius.

Fr. Knapp sagt in seinem Lebrbuche der chemischen Technologie, 3. Auf. I, 218, nachdem er die von Violette ermittelten Resultate ther die Yerkoblung des Holzes bei verschiedenen Temperaturen mitgetheilt hat:

„Es bestehen mithin zwischen Holz und Schwarzkohle Producte, welchedem Wirkungswerthe der letzteren fast gleichkommeñ bei einer nm die Hulfte høberen Ausbeute. Diese Producte sind die sogenannten Rothkohlen und entsprechen einer Verkohlung, bei welcber $60-70$ p.C. vom Gewicht des Holzes ausgetrieben werden. Diese Rothkohlen sind rothbraun von Farbe and Strich and nach Berthier wenig hygroskopisch. Sie haben vielfach die Aufmerksamkeit der Techniker auf sich gezogen, ohne dass jedoch Resultate von bleibendein Werthe daraus hervorgegangen wăren. So einleuchtend nämlich die Sache an sich ist, so gross ist die Schwierigkeit, im Grossen, eine Rothkoble ron einem bestimmten Kohlungszustande in gleichmulssiger Beschaffenheit zu erzengen.

Diese Sehwierigkeit ist als tberwunden $z u$ betrachten, denn der Verein far chemische Industrie in Mainz producirt ein solches Zwischenproduct zwischen Holz und Schwarzkohlen unter dem Namen "Rothholz" schon seit etwa einem Jahre in immer grösseren Quantităten und von so gleichnüssiger Beschaffenheit, wie sich solche bei einem derartigen Verkoblungsproduct tberhaupt nur erwarten liess.

Wie sich aus dem Folgenden ergeben wird, ist die Vorkohlung bei dem Rothbolze nicht ganz so weit getrieben als diess bei den Producten gaschah, welche man bisher als Rothkohle bezeichnete, und es war daher zweckmässig, dass der neuen Ware auch ein neuer Name, das heisst der Name Rothholz, gegeben wurde. 


\section{1) Figenschaften des Rothholzes.}

Das Rothholz aus der genannten Bezugsquelle ist aus Buchenholz dargestellt; es hat, wenn man von der Farbe absieht, noch ganz des Ansehen des Holzes. - Es lässt sich wie dieses spalten, schneiden, sägen, raspeln etc. Der Widerstand, den es beim Zerbrechen leistet, ist jedoch geringer als der des unverkohlten Holzes. Die Farbe ist auf der frischen Spaltfläche vollkommen gleichmässig und zwar glänzend braun, zum Rothbraunen neigend; auf der Aussenseite sind die Scheite etwas dunkler, auch färben diese Aussenflächen etwas ab, wälirend diess bei den Spaltfächen durchaus nicht der Fall ist. Auf Papier macht ein Stift von Rothholz nur bei starkem Aufdrlicken einen schwachen braunen Strich, auf mattem Porzellan einen etwas stärkeren. -

Von Wasser wird das Rothholz nur schwer benetzt, so dass auf eine horizontale Rothholz-Spaltfläche gebrachte Wassertropfen darauf stehen bleiben und nicht wie auf einerBuchenholzspaltfläche bald zerfliessen.

Das specifische Gewicht des Rothholzes, d. h. seine Dichtigkeit als ganzes Stttck, die lufterfullten Zwischenräume mitgerechnet, ist bedeutend geringer, als das des Buchenholzes und beträgt im mittleren Durchschnitt 0,54, während ich das spec. Gew. rollkommen lufttrocknen Buchenscheitholzes zu 0,654 fand.

Die Bestimmungen wurden mit gewogenen Stilcken von etwa $20 \mathrm{Grm}$. ausgeftuhrt, welche einen dünnen Ueberzug von Paraffin erhielten, dessen Gewicht bestimmt wurde. Man ermittelte, wie viel Wasser durch die Sttlcke verdrängt wurde, und nahm natïrlich bei der Berechnung auf das durch die geringe Paraffinmenge verdrängte Wasser Ruicksicht.

Das Rothholz ist sehr wenig bygroskopisch. Ein Stick Rothholz in Gewichte von 34,219 Grm. 48 Stunden lang bei $17^{\circ} \mathrm{G}$. in einen: $z \mathrm{H} 1 / 3 \mathrm{nit}$ Wasser geftullten Kolben so aufgehängt, dass es nicht in das Wasser tauchte, nahm nur um $0,559 \mathrm{Grm}$. zu, somit um 1,63 p.C.

Beim Liegen an der Luft yab das Rothholz das aufgenommene Wasser wieder vollständig $a b$, und schon nach we- 
85 Frcsenius: Das Rothholz aus den Fabriken des Vereins

nigen 'Tagen hatte es sein ursprtingliches Gewicht wieder angenommen.

Iegt man Rotliholz andauernd in Wasser, so nimmt es alluählich eine etwas grössere Menge desselben auf, aber:auch das so aufgesangte wird beim Liegen an der Luft ziemlich rasch wieler abgegeben.

Das Rothholz ist weit entzündlicher als das trockenste Buchenholz. Ein nicht allzu dicker Rothholzspan lässt sich mit Hïlfe eines Zündhölzchens entzlinden und brenut mit Leichtigkeit weiter. Beim Verbrennungsacte entwickelt - das Rothholz zuuächst und relativ rasch eine grosse Masse brennbarer Gasc, welche mit leuchtender Flamme verbrennen, und verwandelt sich in glihende Holzkohlen, welche dann weiter verbrennen.

2) Chemische Zusammensetzung des Rothholzes.

Das frisch dargcstellte Rothholy ist wasserfrei, solches aber, welches längere Zeit hindurch an der Luft gelegen hat, also lufttroekenes Rothholz, enthält eine geringe Menge Wasser. 1,3194 Grm. Rothholz in kleinen Splittern verloren, 10 Stunden hindurch bei $110^{\circ} \mathrm{C}$. getrocknet, 0,0592 Grm., entsprechend 4,4S7 p.C. hygroskopischer Feuchtigkeit (worin 0,4986 p.C. Wasserstoff). Zum Behufe der Elementaranalyse wurde das lufttrockeue Rothholz in reinem Sauerstoffgas verbrannt. Die Verbrennungsproducte strichen über eine lange Schicht gekörnten ylühendeu Kupferoxyd's, bevor sie in die Absorptionsapparate gelangten.

Bei drei Versuchen wurden folgende Zahlen erbalten:

Angewnultex Rothliolz Erhaltene Kohlensänre Erhaltenes Wasser

$\begin{array}{llll}\text { 1) } & 0,1941 & 0,3746 & 0,1070 \\ 2) & 0,203 i & 0,3940 & 0,1140 \\ 3) & 0,3155 & 0,6074 & 0,1839\end{array}$

Daraus berechnen sich folgende Gehalte an Kohlenstoff und Wasserstoff in Procenten:

oder im Mittel :

\begin{tabular}{|c|c|c|c|c|}
\hline & & $\begin{array}{c}1 . \\
52,66\end{array}$ & $\begin{array}{c}11 . \\
52,75\end{array}$ & $\begin{array}{r}\text { HII. } \\
52,51\end{array}$ \\
\hline & & 6,13 & 6,22 & 6,48 \\
\hline
\end{tabular}

Kohlenstoff * $\quad \begin{array}{r}52,64 \\ 6,28\end{array}$ 
Beim Verbrennen in Platintiegel lieferten $25 \mathrm{Grm}$. Rothholz 0,1225 Grm. Asche oder 0,490 p.C.

Diese 0,1225 Grm. Asche enthielten 0,0158 Grm. Kohlensăure, somit betrügt die Kohlenš̌ure in der Asche, bezogen auf 100 'Theile Ruthholz, 0,06321 Theile, entsprechend Kohlenstoff 0,0172 Theile. Dieser in der Asche in Gestalt von Kohlensäure zurückgehaltene Kohlenstoff ist somit dem gefundenen mittleren Kohlenstoffgehalt noch zuzurechnen, während von dem gefundenen Wasserstoff der abzuzieben ist, welcher dem bygroskopischen Wasser angehört, nämlich 0,4986 , weenn der in organischer Substanz vorhandene Wasscrstoff resultiren soll.

Mit Bertlcksichtigung dieser Correcturen ergiht sich alsdann fir das lufttrockene Rothloolz folgende procentische $\mathrm{Zu}$ sammensetzung:

\begin{tabular}{|c|c|c|}
\hline $\begin{array}{l}\text { Kohlenstoff . . . . } \\
\text { Wraserstoff . . . . }\end{array}$ & 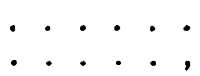 & $\begin{array}{r}52,66 \\
5,7 \mathrm{~s}\end{array}$ \\
\hline Sauterstuff nubst Spuren & n von Stickstoff & 36,64 \\
\hline Kohlensïurefreie Asche & . & 0,43 \\
\hline Wasser & • & 4,40 \\
\hline
\end{tabular}

3) Wurmeeffect des Rothbolzes aus der Flementaranalyse berechnet und verglichen mit dem des luftrockenen Buchenholzes.

Wenn schon es bekannt, duss die Berechnung des Wänneeffects aus der Elementaranalyse der Brennstoffe die Sicherheit nicht bietet, welche man ihr frther beilegte *), so gibt sue doch Annaherungswerthe und vergleichbare Zahlen, wenn die Effecte chemisch ahnlicher Brennstoffe nach denselben Principien berechniet werden. Aus diesem Grunde sollen die so sich ergebenden Wärmeeffecte des Rothbolzes mit denen des lufttrockenen Buchenholzes (Fagus sylvatica) verglichen werden. Das letztere enthalt aschen- und wasserfrei im Mittel der Analysen von Sch bdler und Petersen, von Chevandier und von Baer:

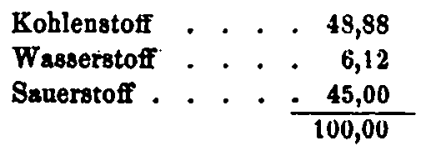

•) Vergl. Kn app a. an O. S. 298 u. f. 
Lufttrockenes Buchenholz, wie es gewöhnlich als Brennmaterial benutzt wird, mit 20 p.C. Wasser und Asche, enthält somit in 100 Theilen:

$$
\begin{aligned}
& \text { Kohlenstoff : . . 39,10 } \\
& \text { Wasserstoff . . . } 4,90 \\
& \text { Sauerstoff . . . . 36,00 } \\
& \text { Wasser und Asche } \cdot \frac{20,00}{100,00}
\end{aligned}
$$

a) Absoluter Warme-Effect.

Da die 36,00 p.C. Sauerstoff, welche das lufttrockene Buchenholz enthält, 4,50 Wasserstoff zur Wasserbildung verlangen, so bleiben fur den Heizeffect nur tubrig die 39,10 p.C. Kohlenstoff, und 0,40 p.C. Wasserstoff, welche, wenn man den absoluten Wärmeeffect des Kohlenstoffs zu 8080 und den des Wasserstoffs zu 34462 annimmt, 3297 Wärmeeinheiten liefern, von denen die abzuziehen sind, welche durch Ueberfuhrung des rorhandenen und erzeugten Wassers in Wasserdampf verloren gehen, nämlich 6,52 (Wasser $+9 / 80)=$ 394 W. E.

Ein Gewichtstheil lufttrockenes Buchenholz ubt somit einen Heizeffect von $2903(\mathrm{C}=8080)$ oder von $0,36(\mathrm{C}=1)$ aus.

Berechnen wir nun den gbsoluten Wärme-Effect des lufttrockenen Rothholzes nach denselben Principien, so erhalten wir 4371 Wärmeeinheiten $(C=8080)$ oder $0,541(C=1)$. Der Wärme-Effect, den man mit gleichen Gevichtstheilen lufttrockenen Buchenholzes und lufttrockénen Rothholzes zu erzielen vermag, verbält sich somit wie 1 (Buchenholz): 1,5 (Rothholz), und man muss somit der Theorie nach 150 Pfund lufttrockenes Bnchenholz aufwenden, um den Effect von 100 Pfund lufttrockenem Rothholz zu erreichen.

b) Specifischer Warme-Effect.

Derselbe repräsentirt bekanntlich die Wärmeimenge, welche ein bestimmtes Volumen eines Brennmaterials bei seiner vollständigen Verbrennung entwickelt, und wird durch das Product ausgedrückt, welches man erhält, wenn man das spec. Gew. des Brennmaterials mit dem absoluten Wärme-Effect multiplicirt. 
Derselbe ist somit für Rothholz:

$$
0,54 \times 4371=2360 \text {. }
$$

Fur lufttrockenes Buchenholz:

$$
0,654 \times 2903=1899 \text {. }
$$

Der Wărmeeffect, den man mit gleichen Raumtheilen lufttrocknen Buchenholzes und Rothholzes zn erzielen vermag, verhält sich somit wie 1 (Buchenholz): 1,243 (Rothholz), und man muss somit der Theorie nach in runden Zahlen $1 \frac{1}{4}$ Stecken oder Klafter lufttrocknes Buchenholz aufwenden, um den Effect von 1 Stecken oder Klafter Rothholz zu erreichen.

c) Pyrometrischer Warme-Effect oder Verbrennungstemperatur.

Denkt man sich, dass 1 Kilogramm lufttrockenes Buchenbolz, von der zuvor angenommenen Zusammensetzung, auf Kosten der gerade nothigen Menge atmosphärischer Luft verbrannt wird, so werden als zu erbitzende Gase erhalten:

$$
\begin{array}{ll}
\text { 1,43 Kilogrm. Kohlensäure, } \\
\mathbf{0 , 6 4} \quad \text { Wasserdampf, } \\
3,58 \quad \text { Stickstoff. }
\end{array}
$$

Multiplicirt man diese Mengen mit der specifischen Wärme der betreffenden Gase (und zwar hahe ich bei den Berechnungen die von de la Roche und Berard ermittelten Werthe zu Grunde gelegt, wonach die specifische Wärme der Kohlensäure 0,221, die des Wasserdampfes 0,847 , die des Stickgases 0,275 und die des Sauerstoffs 0,236 ist), so erbălt man als Summe 1,842, und dividirt man mit dieser in 2903 , den absoluten Wärmeeffect, so erbält man als pyrometrischen Effect des lufttrocknen Buchenholzes $1575^{\circ} \mathrm{C}$.

Geht man dagegen von der Annahme aus, dass noch ein weiteres, dem zum vollständigen Verbrennen theoretisch nothwendigen Volum gleiches Volum atmosphärischer Luft aufgewandt werden mltsse, um den Zweck der vollständigen Verbreunnng wirklich zu erreichen, so sind bei obiger Annahme weiter zu.erbitzen 1,07 Kilogrm. Sauerstoff und 3,58 Stickstoff. Nach Multiplication mit den betreffenden Zahlen fttr die specifische Wärme erhält man alsdann 3,079 und durch Division dieser Zahl in 2903 als pyrometrischen Effect $943^{\circ} \mathrm{C}$. 
Beim Verbrennen von 1 Kilogrm. Rothholz von obiger Zusammensetzung mit der theoretisch nöthigen Luftmenge erhält man als zu erhitzende Gase 1,93 Kilogrm. Koblensäure 0,56 Kilogrm. Wasserdampf und 4,99 Kilogrm. Stickgas. Durch Multiplication mit den beztiglichen die specifische Wärme ausdrtickenden Zahlen erhält man 2,272 und durch Division dieser Zahl in 4371 (den absoluten Wärme - Effect) erhalt man als pyrometrischen Wärme-Effect des Rothholzes $1924^{\circ} \mathrm{C}$.

Bei Anvahme der doppelten Luftmenge sind weiter zuerhitzen 1,5 Kilogrm. Sauerstoff und 4,99 Stickstoff. Nach Multiplication niit den die spec. Wärme ausdrttckenden Zahlen erhält man somit 4,001 und durch Division in 4371 als pyrometrischen Wärme-Effect $1093^{\circ} \mathrm{C}$.

Bei Annahme der zum Verbrennen der Theorie nach nöthigen Luftmenge tibertrifft somit die beim Verbrennen des Rothholzes erzeugte Hitze die beim Verbrennen lufttrocknen Buchenholzes um $349^{\circ} \mathrm{C}$, bei Annahme der doppelten Luftmenge um $150^{\circ} \mathrm{C}$.

Bei der.Unsicherheit, wie viel tiberschüssige Luft factisch mit erhitzt werden muss, um die vollständige Verbrennung eines Brenninaterials herbeizufuhren, und bei der Verschiedenheit der Angaben tiber die spec. Wärme des Wasserdampfs $(0,847$ nach de la Roche und Berard - 0,475 nach Reḡnault) haben die durch solche Berechnungen ermittelten Zahlen nur einen relativ geringen Werth; sie gentigen aber jedenfalls um darzuthun, dass die Temperatur, welche durch Verbrennen von Rothholz auftritt, wesentlich höher ist, als die durch Verbrennen lufttrockenen Buchenholzes zu erzielende.

4) Praktische Versuche, um den Heizwerth des Rothholzes mit dem von lufttrockenem Buchenholze zu vergleichen.

Um den in gewöhnlichen Feuerungen factisch zu erzielenden Heizeffect des Rothholzes mit dem lufttrocknen Buchenholzes zu vergleichen, stellte ich zunächst einen Versuch an unter Benutzung eines viereckigen kupfernen Kessels von $120 \mathrm{Cm}$. Länge, $60 \mathrm{~cm}$. Breite und $25 \mathrm{Cm}$. Tiefe, der in meinem Laboratorium zur Darstellung von destillirtem Wasser und zu- 
gleich (da er eingesetzte Schränkchen enthält) zum Trocknen bei $100^{\circ} \mathrm{C}$. und auf seiner horizontalen Oberfläche zu Digestionen etc. dient.

Die Feuerung befindet sich in der Art unter dem Kessel, dass dessen ganze untere Fläche der Lănge nach von der Flamme bestrichen wird; die Verbrennungsproducte streichen alsdann unter einem Sandbade von $120 \mathrm{Cm}$. Länge,, $60 \mathrm{Cm}$. Breite und $5 \mathrm{Cm}$. Tiefe hin und gelangen erst dann in den Kamin.

Der Kessel wurde zu zwei Drittel mit Wasser geftillt und dieses mit Steinkohlen zum Sieden erhitzt, bis die Destillation in gewöhnlichem Gange war. Man entfernte jetzt die Steinkollen und verbrannte unter dem Kessel nach und nach 30 Pfund besten lufttrocknen Buchenholzes und zwar so, dass die Destillation des Wassers in möglichst gleichmässigem Gange blieb. Nachdem die 30 Pfund Holz verbrannt waren, was nach $\mathbf{A b}$ lauf von 3 Stunden und 25 Minuten der Fall war, wurde das erhaltene destillirte Wasser gemessen; es betrug 22 Liter. Nebenbei พंurde auch die Temperatur des Sandbades bestimmi, welehes von den abgehenden Verbrennungsproducten bestricben warde and zwar mittelst aines Tbermómeters; welches in den Sand eingesetzì war und während dieses und des folgenden Versuches an derselben Stelle eingesetzt blieb.

Nach Ablauf von je 15 Minuten wurde: der Thermometerstand notirt und es ergah sich so fur die ganze Dauer des Versuchs cine mittlere Temperatur des Sandbades von $133,2^{\circ} \mathrm{C}$.

Würden die angewandten 30 Pfund Holz vollständig verbrannt und alle erzeugte Hitze zur Verdampfung von Wasser verwandt worden sein, so hätte - wenn man den oben ermittelten absoluten Wairme-Effect für das bei diesem Versuche angewandte Holz gelten lässt - die Henge des erhaltenen destillirten Wassers 79,2 Liter betragen müssen. Es haben also nur 27,8 p.C. der durch Verbrennnng des Holzes erzeugten Wärme (vollständige Verbrennung vorausgesetzt) zur-Wasserverdampfung gedient, der Rest beizte das Sandbad, den Kamin oder ging dureh Ausstrahlıng mnd Ableitung verloren. 
Der Versuch wurde nun in ganz gleicher Weise wiederholt, aber anstatt der 30 Pfund lufttrocknen Buchenholzes 30 Pfund Rothbolz rerwandt. Mit dieser Menge liess sich die Destillation 4\% Stunden hindurch in sebr gutem Gange erhalten. - Die Menge des destillirten Wassers betrug 40,5 Liter, die mittlere Temperatur des Sandbades $112,1^{\circ} \mathrm{C}$. Der Theorie nach batten mit 30 Pfund Rotbholz unter den beim Holze angefuhrten Voraussetzungen 119,25 Liter Wasser verdamptt werden können, somit haben von der beim Verbrennen des Rothholzes im Ganzen erzeugten Warme 34 p.C. zur Wasserverdampfung gedient, der Rest zar Heizung des Sandbades, Kamins etc.

Es verhalt sich somit die Verdampfangskraft des lufttrocknen Buchenholzes zu der des Rothholzes, bei dem benutzten Destillirapparate, wie 54,32 : 100.

Ein zweiter analoger Versuch wurde mit einer ronden Destillirblase vorgenommen, deren Zinnhelm abgenommen war, so dass das Wasser frei verdampfen konnte.

Der Kessel mit Wasser von $100^{\circ}$ C. zu zwei Drittel geftllt, wurde bedeckt gewogen und dann 10 Pfand luftrocknes Buchenbolz darunter verbrannt, so dass das Wasser stets im Sieden blieb. Der Versuch dauerte $i 1 / 2$ Stunden. Der Kessel wurde nunmehr wieder bedeckt und gewogen; es fand sich, dass 13 Pfund Wasser verdampft waren. Derselbe Versuch mit 10 Pfund Rothholz dauerte 2\% Stunden und lieferte eine Wasserverdampfung von 24 Pfund. Es verhielt gich somit auch bei diesem Apparate die Verdainpfungskraft des lufttrocknen Buchenholzes zu der des Rothholzes fast genau wie bei dem vorigen, nămlich wie 54,17: 100.

Man erkennt somit, dass der bei verschiedenen Feuerungen praktisch sich ergebende Heizeffect, dem des Holzes gegentber, sich noch weit gunstiger stellt, als diess die Vergleichung der absoluten Warmeeffecte erschliessen lisst, und 2war offenbar desshalb, weil in den geworhnlichen Feuerungen vollstandige Verbrennung bei Holz schwerer zu erreichen ist als bei Rothbolz, und weil zweitens bei der an Wasserdampf reichen Holzflamme unter sonst gleichen Verhaltnissen eine weit grobsere Warmemenge in den Schornstein gelangt als beim Verbrennen 
des Rothholzes, wie man diess aus der Vergleichung der Sandbadtemperaturen bei den erstgenannten Versuchen recht deutlich ersieht, denn die Sandbaderbitzung repräsentirt ja gewissermassen schon verlorene Wärme.

\section{5) Praktische Verwendung des Rothholzes.}

Da der Verein fur chemische Industrie in Mainz 1 Vol. Rothholz zu demselben Preise liefert, welchen 1 Vol. lufttrockenes Buchenholz hat, so ergiebt sich zunächst, dass das Rothholz ein billigeres Brennmaterial ist als das Buchenholz, weil man bei seiner Verwendung mit gleichem Geldaufwande weit grösseren Heizeffect erzielen kann. Gegenwärtig kostet in Mainz, frei ins Haus geliefert, 1 Vol. geschnittenes Buchenbolz gleich 1200 Pfund $11 \mathrm{fl}$., und ebenso viel, frei ins Haus geliefert, ein gleiches Volum Rothholz gleich 920 Pfund.

Nach diesen Preisen hätten die beiden ersten in 4 genannten Versuchen aufgewandten 30 Pfund Holz, womit 22 Liter destillirtes Wasser erbalten wurden, 16,5 Kreuzer, und die 30 Pfund Rothholz, welche 40,5 Liter Wasser lieferten 21,5 Kreuzer gekostet; somit kommen auf 10 Liter destillirtes Wasser beim Brennen ron Buchenholz 7,5 Kreuzer, beim Brennen von Rothholz dagegen nur 5,3 Kreuzer.

Das Rothbolz ist ferner ein äusserst bequemes Brennmaterial, weil es sich so leicht entzünden lässt, dass das Anmachen von Feuer jeder Art mit Hülfe desselben ausserordentlich leicht ist, und weil man bei Verwendung in eisernen Oefen oder Heerden ein kleines Scheit nach dem andern anlegen kann, ohne furchten zu missen, dass das Feuer erlischt. Seine Leichtentzundlichkeit und die Raschheit, mit der es ohne weiteres in den vollen Verbrennungsprocess eintritt, macht es namentlich in solchen Fällen ausserordentlich angenehm, in denen man die Feuerwirkung, sei es in Heerden oder Oefen, gesch wind herbeizuftihren wïnscht, und die Gleichmässigkeit seines Verbrennens lässt es namentlich für Thonund sogenannte Porcellanöfen sehr geeignet erscheinen, weil bei diesen der Zeitpunkt, bei welchem die Ofenthüre zugeschraubt, beziehungsweise die Klappe geschlossen werden kann sehr leicht und sicher zu treffen ist. 
Das Roth holz einpfiehlt sich auch als leitht transportabeles Brennmaterial, sei es im Hinblick auf Eisenbahn- oder sonstigen Achsen-Transport, sei es in Betreff des Transports aus dem Holzstall oder Keller in Kuche und Zimmer, sofern bei gleichem factiseh zur Verwendung kommenden Heizeffect das dazu erforderliche Rothbolz kaum wehr als halb so riel wiegt, als Buchenbolz. Das Rotlbolz ist weiter ein sehr hafibares und leicht aufzubewahtendes Brennmaterial, da es dem Vermodern und Verstocken gar nicht auggesetzt, und sehr wenig bygroskopisch ist.

Es kaun daher ebensogut im Keller wio in Holzstalle aufbewahrt werden, und selbsit solches, welches im Freien liegt, und vou Rogen ganz durchnsset ist, erscheint scbon nach wenigen trocknen Tagen zum Brennen wieder wohl geeignet.

Das Rothbolz ist endlich als intensive Iitze erzeugendes Brennmaterial fur viele industrieile Zweeke höchst geeignet und zwar aberall da, wo man auf Holsfeucrung angewiesen ist und holer Hitzgrade bedar.

Diese liefert weit besser und sicherer als dus beste gedörrte Hols, und es eignet sich daher das Roth holz nanientlich auch zur Verwendung in Brennofen fitr Porzellau etc.

Auch die Eisedultteniadustrie kana daron groseen Vortheil ziehen, da die Erfulhung bereits mit voller Sicherheit. dargethan bat, dass beirn Holzkohleu - Hochofenbetrieb ein gewisser'Theil der Holzkohlen durch klein geschnittenes Rothholz mit erbeblicher Kustencrsparuny ersetzt werden kaon.

Wiesbaden, den 16. Jan. 1868. 